\title{
Global stellar magnetic fields: the crossover from fossils to dynamos
}

\author{
Gautier Mathys \\ European Southern Observatory, Casilla 19001, Santiago 19, Chile \\ email: gmathys@eso.org
}

\begin{abstract}
Ap stars comprise the majority of early-type stars in which magnetic fields have been definitely detected. Their observational properties are reviewed, with emphasis on those relevant to the understanding of the origin and evolution of their fields.
\end{abstract}

Keywords. Stars: magnetic fields, stars: early-type, stars: chemically peculiar

While the presence of magnetic fields is ubiquitous in lower main sequence stars, only about $10 \%$ of early-type stars are observably magnetic. Most of them are main-sequence A- and B-type stars in the atmospheres of which a number of chemical elements are strongly over- or under-abundant. These Ap and Bp stars (often conveniently referred to collectively as Ap stars) generally show periodic variations of one or more of their observed properties: photometry, spectral line intensities, and magnetic fields. They are explained by the Oblique Rotator Model, in which the aspect of the visible stellar hemisphere changes as the star rotates because the distribution of the elemental abundances, brightness and magnetic field over the stellar surface is not symmetric about its rotation axis. Hence the variation period is the rotation period of the star. Rotation periods of Ap stars range from half a day to several decades: Ap stars rotate in average slower than superficially normal A- and B-type dwarfs in the same temperature range. For more details, see Mathys (2004a).

Magnetic fields of Ap stars cover their whole surface and show a considerable degree of large-scale organization; their strength varies from place to place on the star within a limited range. In first approximation, their structure often resembles a single dipole. The properties of Ap star magnetic fields have been recently reviewed by Mathys (2004b); here I shall only mention some of them, with emphasis on their relevance for the understanding of the origin and evolution of the fields. For a more complete recent presentation of the theoretical studies of the latter, see Moss (2004).

The favoured (albeit not undisputed) view is that the large-scale magnetic fields of the Ap stars have a fossil origin: they are either the relic of a field present in the interstellar medium at the time of star formation, and somehow locked into the star when it contracted, or the relic of a pre-main sequence dynamo. One merit of the fossil field hypothesis is that it provides a natural explanation of the fact that fields are observed in only a fraction of the early-type stars, and of the co-existence of observably magnetic and non-magnetic stars in the same environment (e.g., binaries, clusters). It has long been questioned, though, whether field configurations with sufficient long-term stability are possible: recent theoretical work by Braithwaite \& Spruit (2004) and by Braithwaite \& Nordlund (2006) now appears to have solved this difficulty.

The evolutionary status of magnetic Ap stars, and in particular the stage at which they become observably magnetic, has been hotly debated in recent years. There now seems to be some consensus emerging, according to which magnetic Ap stars with $M>3 \mathrm{M}_{\odot}$ appear homogeneously distributed across the whole width of the main sequence band, 
while lower mass magnetic Ap stars tend to be concentrated around the centre of this band (Hubrig et al. 2000; Kochukhov \& Bagnulo 2006). This concentration may possibly be explained by the secular evolution of stable field configurations (Braithwaite \& Nordlund 2006).

For a long time, observers have tried to identify the progenitors of magnetic Ap stars. The latter may finally have been found with the recent detections of magnetic fields in Herbig Ae/Be and related stars by Hubrig et al. (2004) and by Wade et al. (2005).

More than $10 \%$ of the Ap stars whose periods (or their lower limits) have been determined have periods longer than 100 days (Mathys 2004a). This leaves little doubt that these long periods are genuinely part of the Ap phenomenon, and that any theory describing how Ap stars form and acquire their properties must be able to account for them. Convincing arguments have been presented supporting the view that the variations of the Ap stars with extremely long periods do result from rotation, like for their shorter period counterparts. Furthermore, differences in the magnetic properties between extremely slowly rotating and faster rotating Ap stars have been identified. One of them is based on consideration of the mean magnetic field modulus, that is, the average over the visible stellar hemisphere of the modulus of the magnetic vector, weighted by the local emergent line intensity. This field moment can be measured only in those stars that rotate slowly enough and that have a sufficiently strong field so that their spectral lines can be observationally resolved into their Zeeman split components. At present, about 50 such stars are known, of which approximately $50 \%$ have a rotation period longer than $150 \mathrm{~d}$. None of these long-period stars have a mean field modulus exceeding $7.5 \mathrm{kG}$, while in more than half of the stars with periods shorter than 150 days, the mean field modulus is greater than $7.5 \mathrm{kG}$ (Mathys et al. 1997). On the other hand, Landstreet \& Mathys (2000) and Bagnulo et al. (2002), using models with a predominant dipole component to represent the structure of Ap star magnetic fields, showed that in stars with periods shorter than $30 \mathrm{~d}$, the angle between the dipole axis and the stellar rotation axis is generally large, while the two axes are nearly aligned in longer-period stars. This result may possibly be explained by the accretion braking mechanism proposed by Mestel \& Moss (2005).

In summary, while in recent years significant progress has been achieved in the understanding of the origin and evolution of the magnetic fields of early-type stars, many questions remain open, which require further observational and theoretical studies.

\section{References}

Bagnulo, S., Landi Degl'Innocenti, M., Landolfi, M., \& Mathys, G. 2002, A\&\&A, 394, 1023

Braithwaite, J., \& Nordlund, Å. 2006, A\& A, 450, 1077

Braithwaite, J., \& Spruit, H. C. 2004, Nature, 431, 819

Hubrig, S., North, P., \& Mathys, G. 2000, ApJ, 539, 352

Hubrig, S., Schöller, M., \& Yudin, R. V. 2004, A\&A (Letters), 428, L1

Kochukhov, O., \& Bagnulo, S. 2006, A\&A, 450, 763

Landstreet, J. D., \& Mathys, G. 2000, A\&A, 359, 213

Mathys, G. 2004a, in: A. Maeder \& P. Eeenens (eds.), Stellar Rotation, Proc. IAU Symp. No. 215

(San Francisco: ASP), p. 270

Mathys, G. 2004b, in: J. Zverko, J. Žižňovský, S. J. Adelman \& W. W. Weiss (eds.), The A-Star Puzzle, Proc. IAU Symp. No. 224 (Cambridge: CUP), p. 225

Mathys, G., Hubrig, S., Landstreet, J. D., Lanz, T., \& Manfroid, J. 1997, A\&AS, 123, 353

Mestel, L., \& Moss, D. 2005, MNRAS, 361, 595

Moss, D. 2004, in: J. Zverko, J. Žižňovský, S. J. Adelman \& W. W. Weiss (eds.), The A-Star Puzzle, Proc. IAU Symp. No. 224 (Cambridge: CUP), p. 245

Wade, G. A., Drouin, D., Bagnulo, S., et al. 2005, A\&A (Letters), 442, L31 